\title{
Machine Learning for Energy Systems
}

\author{
Denis Sidorov ${ }^{1,2, *(\mathbb{D})}$, Fang Liu ${ }^{3}(1)$ and Yonghui Sun ${ }^{4}$ \\ 1 Applied Mathematics Department, Energy Systems Institute, Siberian Branch of Russian Academy of \\ Sciences, 664033 Irkutsk, Russia \\ 2 Industrial Mathematics Laboratory, Baikal School of BRICS, Irkutsk National Research Technical University, \\ 664074 Irkutsk, Russia \\ 3 School of Automation, Central South University, Changsha 410083, China; csuliufang@csu.edu.cn \\ 4 College of Energy and Electrical Engineering, Hohai University, Nanjing 210098, China; \\ sunyonghui168@gmail.com \\ * Correspondence: dsidorov@isem.irk.ru; Tel.: +7-3952-500-656 (ext. 258)
}

Received: 5 August 2020; Accepted: 1 September 2020; Published: 10 September 2020

\begin{abstract}
The objective of this editorial is to overview the content of the special issue "Machine Learning for Energy Systems". This special issue collects innovative contributions addressing the top challenges in energy systems development, including electric power systems, heating and cooling systems, and gas transportation systems. The special attention is paid to the non-standard mathematical methods integrating data-driven black box dynamical models with classic mathematical and mechanical models. The general motivation of this special issue is driven by the considerable interest in the rethinking and improvement of energy systems due to the progress in heterogeneous data acquisition, data fusion, numerical methods, machine learning, and high-performance computing. The editor of this special issue has made an attempt to publish a book containing original contributions addressing theory and various applications of machine learning in energy systems' operation, monitoring, and design. The response to our call had 27 submissions from 11 countries (Brazil, Canada, China, Denmark, Germany, Russia, Saudi Arabia, South Korea, Taiwan, UK, and USA), of which 12 were accepted and 15 were rejected. This issue contains 11 technical articles, one review, and one editorial. It covers a broad range of topics including reliability of power systems analysis, power quality issues in railway electrification systems, test systems of transformer oil, industrial control problems in metallurgy, power control for wind turbine fatigue balancing, advanced methods for forecasting of PV output power as well as wind speed and power, control of the AC/DC hybrid power systems with renewables and storage systems, electric-gas energy systems' risk assessment, battery's degradation status prediction, insulators fault forecasting, and autonomous energy coordination using blockchain-based negotiation model. In addition, review of the blockchain technology for information security of the energy internet is given. We believe that this special issue will be of interest not only to academics and researchers, but also to all the engineers who are seriously concerned about the unsolved problems in contemporary power engineering, multi-energy microgrids modeling.
\end{abstract}

Keywords: industrial mathematics; pattern recognition; inverse problems; intelligent control; artificial intelligence; energy management system; smart microgrid; energy systems; forecasting; optimization; Volterra equations; energy storage; load leveling; power control; offshore wind farm; cyber-physical systems 


\section{Introduction}

Future energy systems will grow in complexity, causing both higher demands in reliability and an increase in the degrees of freedom for functional improvement of integrated multi-energy systems. Progress in mathematical modeling tools development based on heterogeneous data acquisition, data fusion, cybersecurity, and global navigation satellite systems (GNSS) opens new perspectives in modern energy systems rethinking and improvement.

Machine learning-based data-driven models have exceptional potential to play the important role of improving the comprehensive utilization rate of multi-energy including renewables. With the wide interconnection of source-storage-load equipment at the multi-energy smart grid level through wired/wireless communication networks, the multi-energy grid has gradually evolved into a highly coupled cyber-physical system, and the traditional operation and control methods are difficult to apply.

This Special Issue of Energies aims at addressing the top challenges in energy systems development, including electric power systems, heating and cooling systems, and gas transportation systems. Special attention is paid to the efficient mathematical methods integrating data-driven black box dynamical models with classic mathematical and mechanical models and methods.

\section{Brief Overview of the Contributions}

The work "A New Hybrid Short-Term Interval Forecasting of PV Output Power Based on EEMD-SE-RVM" [1] by S. Wang et al. proposed a novel hybrid model for short-term PV output power interval forecasting based on sample entropy, ensemble empirical mode decomposition (EEMD), and relevance vector machine (RVM). The PV output power sequences were decomposed into several intrinsic mode functions (IMFs) and residual components by EEMD. The frequency domain decomposition helped to reduce the influence of noise. Then, the SE algorithm was utilized to reconstruct the components, with typical characteristics, into trend decomposition and detail decomposition which were prepared for point forecasting and interval forecasting, respectively. After that, the forecasting results were superimposed for the overall forecasting results. The simulation results verified the proposed hybrid model. The conclusion suggested that the proposed hybrid model improved both the reliability and sharpness of prediction intervals, and it was suitable for practical application on other renewable energies output power forecasting.

F. Liu, R.R. Li, and A. Dreglea in the work titled "Wind Speed and Power Ultra Short-Term Robust Forecasting Based on Takagi-Sugeno Fuzzy Model" [2] proposed an ultra short-time forecasting method based on the Takagi-Sugeno (T-S) fuzzy model for both wind power and wind speed. First, a fuzzy C-means (FCM) algorithm was utilized to cluster the dataset. Then, the T-S fuzzy model was studied for ultra-short-term forecasting. Then, the recursive least squares (RLS) algorithm was used to quantify the consequent parameters of the T-S fuzzy model. The comparison results showed that the proposed method had higher accuracy, compared to the existing methods. The conclusion suggested that the errors of proposed method were smaller. Meanwhile, the proposed method also handled mutation points better.

S.F. Stefenon and R.Z. Freire et al. in the work titled "Electrical Insulator Fault Forecasting Based on a Wavelet Neuro-Fuzzy System" [3] presented the novel approach for predicting electrical insulator conditions. An offline time series forecasting approach with an adaptive neuro-fuzzy inference system (ANFIS) was studied. Then, wavelet packets transform (WPT) was associated to the ANFIS model for the improvement of time series forecasting performance and the noise reduction. Besides, distinct parameters were adjusted to improve the model performance. The numerical comparisons were presented to verify the effectiveness of the proposed methods. The conclusion suggested that ANFIS was a reasonable approach, considering both computational effort and performance.

In the work "Cluster-Based Prediction for Batteries in Data Centers" [4] S.N. Haider et al. proposed a clustered auto-regressive integrated moving average (ARIMA) for forecasting battery's health. The clustering approaches were studied to obtain the accurate patterns in data sets for the improvement of ARIMA. The numerical results verified the performance of the proposed method. The conclusion 
suggested that the clustered ARIMA had better performance, compared to the single predictor and total data predictors. Meanwhile, the k-shape-based clustering assisted results were more accurate than the dynamic time warping clustering. It is to be noted that the efficient maintenance of storage systems is one of the corestones for future power systems and these studies will support such systems developent.

In the work titled "An Integrated Methodology for Rule Extraction from ELM-Based Vacuum Tank Degasser Multiclassifier for Decision-Making" [5] S.H. Wang et al. proposed a method of rules extraction from the trained extreme learning machine (ELM) classification model for the decision-making purposes. First, a three-class classification problem of the end temperature in the vacuum tank degasser (VTD) system was studied. Second, an ELM multiclassifier was studied to instruct the end temperature in different ranges. Finally, based on the classified training data set, rules were extracted with discrete and continuous features utilizing the classification and regression trees (CART) algorithm. The experimental results demonstrated the effectiveness of the proposed method. The conclusion suggested that the proposed method was able to classify the end temperature demonstrating the high potential for reliable prediction of the end temperature in a VTD system.

The work titled "Electric Power System Operation Mechanism with Energy Routers Based on QoS Index under Blockchain Architecture" [6] by G.J. Gong et al. proposed an integrated application of blockchain technology on energy routers at transmission and distribution networks with increased renewable energy penetration. This paper studied the operations of energy routers for transmission and distribution networks with high permeability renewable energy access, and the application of blockchain technology integrating the energy flow quality of service index with the independent cooperative mode of the energy router node. Then, the QoS index of energy flow control and energy router node doubly-fed stability control model were designed. Besides, multiobjective particle swarm optimisation (MOPSO) to optimize output of multi-energy power generation was studied. Moreover, in order to resolve those complications in the power mutual aid of energy nodes at all levels, this paper utilized an autonomous energy collaborative optimization mechanism and control process of the router nodes at the transmission and distribution network with the blockchain as the technical support. Finally, optimization mechanism and control flow of autonomous energy coordination of b2u (bottom-up) between router nodes of transmission and distribution network were studied. The simulations verified the effectiveness of the proposed methods.

The work conducted by Z.L. Zeng et al. [7] titled "Blockchain Technology for Information Security of the Energy Internet: Fundamentals, Features, Strategy and Application" first studied the information security problems existing in the energy internet from system control layer, device access, market transaction and user privacy. Then, the multilevel and multichain information transmission model for the weak centralization of scheduling and the decentralization of transaction were proposed. Besides, the information transmission model which was able to solve some of the information security issues was studied. The analysis of applications verified the effectiveness of the proposed blockchain based method. The conclusion suggested that the biggest advantage of the blockchain in information security was its ability to prevent tampering, and it was very difficult for an ordinary information attacker to possess such powerful computing power.

The work by H. Liu et al. [8] titled "Operational Risk Assessment of Electric-Gas Integrated Energy Systems Considering $N-1$ Accidents" proposed a comprehensive energy risk assessment index and a risk assessment strategy for the multi-energy electric-gas integrated energy system (EGIES) considering component $N-1$ accident. Then, the EGIES steady-state analysis model considering the operation constraints was studied to analyze the operation status of each component. After that, the EGIES component accident set was studied to simulate the accident consequences caused by the failure of each component to EGIES. Besides, to identify the vulnerability of EGIES components, EGIES risk assessment system was studied. Then, the risk assessment of IEEE14-NG15 system was constructed. The simulations verified the effectiveness of proposed method. The conclusion suggested that the proposed method was able to assess the coupling and interaction effects between subsystems, 
reflect the security of system operation to a certain extent, and provide scientific decision basis for relevant personnel.

The work by D. Sidorov et al. [9], “Toward Zero-Emission Hybrid AC/DC Power Systems with Renewable Energy Sources and Storages: A Case Study from Lake Baikal Region", proposed the dynamical models of AC/DC hybrid isolated power system consisting of four power grids with renewable generation units and energy storage systems based on deep reinforcement learning and integral equations. The proposed method was based on two-level optimization technique for operational and emergency control of a hybrid AC/DC community. Based on deep reinforcement learning, the optimal energy management policies at the local level of every grid using advanced stochastic optimization method were studied. Meanwhile, the optimal redistribution of active power between subsystems by minimizing network losses was analyzed. The numerical analysis demonstrated the effectiveness of proposed framework. Besides, the conclusion also demonstrated the disadvantages of proposed method which was the future work. Such studies will help to design future multi-energy microgrids and support sustainable development.

The work by R.Y. Zhao et al. [10], "An Improved Power Control Approach for Wind Turbine Fatigue Balancing in an Offshore Wind Farm", proposed an improved power control approach to optimize the wind turbine (WT) fatigue distribution by balancing the turbulence loads to individual WTs. Then, a control topology was constructed to describe the logical states of the wind farm main controller (WFMC). The simulation results verified that the improved power dispatch approach was able to reduce the mean turbine fatigue of an offshore wind farm, balance the fatigue loads on WTs, further extend the WT lifetime, and reduce the potential maintenance costs.

The work by A.N. Hassan et al. [11], "Two-Layer Ensemble-Based Soft Voting Classifier for Transformer Oil Interfacial Tension Prediction", studied a two-layered soft voting-based ensemble model to predict the interfacial tension (IFT). The performances of multiple machine learning algorithms (as individuals and combined) to predict the transformer oil IFT were also studied. The comparison results revealed that no single technique showed superior performance on all employed metrics. Moreover, the combining methods had better performances. Besides, it was found that feature selection helped to obtain better performance.

The work by R.X. Yang et al. [12], "A Harmonic Impedance Identification Method of Traction Network Based on Data Evolution Mechanism", proposed an identification method based on a data evolution mechanism to improve the identification accuracy of harmonic impedance. The harmonic impedance model and the equivalent circuit of the traction network were firstly studied. Then, the data evolution mechanism based on the sample coefficient of determination was studied to divide results into several reliability levels. In the data evolution mechanism through adding new harmonic data, the high-reliability results covered all frequencies, which improved the accuracy of identification. The simulation results verified the effectiveness of proposed method. The conclusion suggested the proposed method was able to improve the accuracy, but it was mainly used for offline analysis. The computation time and data amount also should be focused on.

The work by F.L. Zhou et al. [13], "Method for Estimating Harmonic Parameters Based on Measurement Data without Phase Angle", proposed a method for estimating harmonic parameters in the case of monitoring data without phase, based on the partial least square regression method. The proposed method utilized the amplitude information of the harmonic voltage and current of the point of common coupling to estimate the harmonic parameters and the harmonic responsibility of each harmonic source. The effectiveness of the proposed method was verified through the simulations. The conclusion also suggested that the background harmonics were able to affect the estimation ability of the algorithm, and it was meaningful to improve the robustness of the algorithm in the future research. 
The work by C.T. Lee et al. [14], "Abnormality Detection of Cast-Resin Transformers Using the Fuzzy Logic Clustering Decision Tree", proposed a fuzzy logic clustering decision tree to diagnose the partial discharges concerning the abnormal defects of cast-resin transformers. Meanwhile, the proposed method integrated a hierarchical clustering scheme with the decision tree to improve the performance. The testing results demonstrated the performance of proposed method. The conclusion demonstrated that the proposed method was able to serve as an effective abnormality detection of cast-resin transformers where real-time processing of data was required. Meanwhile, the future research would focus on the application of the proposed method to resolve complicated fault detection problems.

\section{Concluding Remarks and Outlook}

The Special Issue Book "Machine Learning for Energy Systems" presents a collection of articles dealing with relevant topics in the broad field of data-driven methods. Various mathematical and computational techniques and approaches were presented focusing on different aspects of energy systems. However, all approaches had the computational intelligence and advanced mathematical models at their core. The success of this Special Issue has motivated the editor to propose a new Special Issue that will complement the present one with focus in cyber-physical systems. We invite the research community to submit novel contributions covering how cyber-physical systems and data driven methods can help in improve the future energy systems.

Funding: The reported editorial study was funded by NSFC and RFBR according to the research project No. 61911530132/19-58-53011.

Conflicts of Interest: The authors declare that there is no conflict of interest.

\section{References}

1. Wang, S.; Sun, Y.H.; Zhou, Y.; Mahfoud, R.J.; Hou, D.C. A New Hybrid Short-Term Interval Forecasting of PV Output Power Based on EEMD-SE-RVM. Energies 2020, 13, 87. [CrossRef]

2. Liu, F.; Li, R.R.; Dreglea, A. Wind Speed and Power Ultra Short-Term Robust Forecasting Based on Takagi-Sugeno Fuzzy Model. Energies 2019, 12, 3551. [CrossRef]

3. Stefenon, S.F.; Freire, R.Z.; Coelho, L.D.; Meyer, L.H.; Grebogi, R.B.; Buratto, W.G.; Nied, A. Electrical Insulator Fault Forecasting Based on a Wavelet Neuro-Fuzzy System. Energies 2020, 13, 484. [CrossRef]

4. Haider, S.N.; Zhao, Q.C.; Li, X.L. Cluster-Based Prediction for Batteries in Data Centers. Energies 2020, 13, 1085. [CrossRef]

5. Wang, S.H.; Li, H.F.; Zhang, Y.J.; Zong, Z.S. An Integrated Methodology for Rule Extraction from ELM-Based Vacuum Tank Degasser Multiclassifier for Decision-Making. Energies 2019, 12, 3535. [CrossRef]

6. Gong, G.J.; Zhang, Z.N.; Zhang, X.Y.; Mahato, N.K.; Liu, L.; Su, C.; Yang, H.X. Electric Power System Operation Mechanism with Energy Routers Based on QoS Index under Blockchain Architecture. Energies 2020, 13, 418. [CrossRef]

7. Zeng, Z.L.; Li, Y.; Cao, Y.J.; Zhao, Y.R.; Zhong, J.J.; Sidorov, D.; Zeng, X.C. Blockchain Technology for Information Security of the Energy Internet: Fundamentals, Features, Strategy and Application. Energies 2020, 13, 881. [CrossRef]

8. Liu, H.; Li, Y.; Cao, Y.J.; Zeng, Z.L.; Sidorov, D. Operational Risk Assessment of Electric-Gas Integrated Energy Systems Considering N-1 Accidents. Energies 2020, 13, 1208. [CrossRef]

9. Sidorov, D.; Panasetsky, D.; Tomin, N.; Karamov, D.; Zhukov, A.; Muftahov, I.; Dreglea, A.; Liu, F.; Li, Y. Toward Zero-Emission Hybrid AC/DC Power Systems with Renewable Energy Sources and Storages: A Case Study from Lake Baikal Region. Energies 2020, 13, 1226. [CrossRef]

10. Zhao, R.Y.; Dong, D.H.; Li, C.L.; Liu, S.; Zhang, H.; Li, M.Y.; Shen, W.Z. An Improved Power Control Approach for Wind Turbine Fatigue Balancing in an Offshore Wind Farm. Energies 2020, 13, 1549. [CrossRef]

11. Hassan, A.N.; El-Hag, A. Two-Layer Ensemble-Based Soft Voting Classifier for Transformer Oil Interfacial Tension Prediction. Energies 2020, 13, 1735. [CrossRef]

12. Yang, R.X.; Zhou, F.L.; Zhong, K. A Harmonic Impedance Identification Method of Traction Network Based on Data Evolution Mechanism. Energies 2020, 13, 1904. [CrossRef] 
13. Zhou, F.L.; Liu, F.F.; Yang, R.X.; Liu, H.R. Method for Estimating Harmonic Parameters Based on Measurement Data without Phase Angle. Energies 2020, 13, 879. [CrossRef]

14. Lee, C.T.; Horng, S.C. Abnormality Detection of Cast-Resin Transformers Using the Fuzzy Logic Clustering Decision Tree. Energies 2020, 13, 2546. [CrossRef] 